\title{
PSYCHOMETRIC ANALYSIS OF THE SLOVAK VERSION OF THE POSTPARTUM BONDING QUESTIONNAIRE FOR NON-CLINICAL SAMPLE
}

\author{
Katarína Greškovičová ${ }^{1}$, Barbora Zdechovanová ${ }^{2}$ \& Rebeka Farkašová ${ }^{2}$ \\ ${ }^{I}$ Institute of Applied Psychology, Faculty of Social and Economic Sciences, \\ Comenius University in Bratislava (Slovakia) \\ ${ }^{2}$ Faculty of psychology, Paneuropean University (Slovakia)
}

\begin{abstract}
Bonding represents an emotional tie that one experiences towards one's own child. There are several instruments to measure the level and quality of bonding. Among them we chose and translated the Postpartum Bonding Questionnaire by Brockington et al. (2001) into Slovak language. The aim of this study was to analyse its psychometric qualities. Our non-clinical sample consisted of women $(N=372) 18$ and 44 years $(M=29.74 ; S D=5.25)$ who recently gave birth in Slovakian hospitals. Data collection was carried out from September 2015 until March 2018. Participants filled the Postpartum bonding questionnaire by Brockington et al. (2001) and some of them other three tools: Edinburgh Postnatal Depression Scale by Cox, Holgen and Sagovsky (1987), Depression Anxiety Stress scale-42 (Lovibond \& Lovibond, 1995) and Parental Stress Scale (Berry \& Jones, 1995). The distributions of the items of the bonding were mostly skewed and leptokurtic. Internal consistency is high for the overall Lack of Bonding $(\alpha=.897)$ and varies in factors- $\alpha=.820$ for Impaired Bonding, $\alpha=.779$ for Rejection and Anger, $\alpha=.506$ for Anxiety about Care and $\alpha=.321$ for Risk of Abuse. In order to prove convergent validity, we correlated overall Lack of Bonding with depression (Edinburgh Postnatal Depression Scale, $r s=.251$, Depression Anxiety Stress scale-42 depression rs $=.404 ; n=79$ ), stress and anxiety (Depression Anxiety Stress scale-42, stress $r s=.392$; anxiety $r s=.496 ; n=79$ ) and parental stress (Parental Stress Scale score; $\mathrm{rs}=.674, \mathrm{n}=99$ ). We did not confirm original factor structure via confirmatory factor analysis using principal axis factoring with oblimine rotation. Then, we used principal component analysis with varimax rotation method to reduce the items. 6 components were extracted. Component 1 was comprised of 15 items that explained $35,6 \%$ of the variance with loadings from .306 to .733 . Hence, we proposed new item-structure for the Slovak PBQ. We concluded that the Slovak version of the PBQ proved to have good overall reliability. We found evidences for the convergent validity with parental stress, anxiety, stress, and partly depression, because there were two different results. We also suggest creating a shorter version based on the analysis. Among limits we can see tools used for validity evidence and sample without participants for clinical population. We advise to use the Slovak version of the Postpartum Bonding Questionnaire as a tool to measure bonding in a research context and to use overall summary index (Lack of Bonding) instead of factors.
\end{abstract}

Keywords: Bonding, postpartum bonding questionnaire, psychometric analysis, women.

\section{Introduction}

Mother-infant relationship or bonding is difficult to examine and ambiguity of the concept results in various definitions and tools to measure it (Perrelli et al., 2014; van Bussel et al., 2010). Greškovičová (2016) specify postpartum bonding as an emotional bond that is a result of two aspects of parental care system: external and internal. External aspect of bonding represented by behavioral schemas (e.g. holding, carrying, rocking, feeding) is visible and subject to observation, while internal aspect subsumes an affective (emotions of a caregiver towards a child) and cognitive compound (attitudes of a caregiver towards a child and mental representations of these interactions). Observational or other qualitative methods to investigate postpartum bonding are time-consuming, skills and ability demanding. Hence, self-reported tools seem much more feasible while saving time and personnel sources. They study internal aspect of the bonding (Greškovičová, 2016) and aim to catch not only the quantity but also the quality of the relationship. The questions or items in the bonding questionnaires relate mainly to the 
mother's own perception of her emotional and cognitive responses to the infant (Brockington et al., 2001; Taylor et al., 2005; Wittkowski et al., 2007).

Since in Slovakia there is still absence of tools to tap mother-infant relationship, we translated the Postpartum Bonding Questionnaire created by psychiatrist Brockington and his team (Brockington et al., 2001) several years ago (Greškovičová, 2016). It has been mostly and widely used tool and has been translated into many different languages (Perrelli et al., 2014). It serves to detect early indications of maternal-child disorders, is used in the first weeks after birth and its design and items formulation fall into the internal aspect of the bonding as stated by Greškovičová (2016). Originally the PBQ items were selected by principal component analysis from a pool of 84 items identified by professionals in the clinical field. Both four components and summary index (lack of bonding) are used in clinical and research practice. Whilst this overall summary index appears to be valid and reliable, later studies and cross-cultural validations have shown other factorial structure (one, three, four, but different to the original one) (Ghahremani et al., 2019). With regard to inconsistent and conflicting results of the factor solution of the PBQ and absence of a proper instrument in Slovakia, it motivated us to scrutinize psychometric properties of the tool. Therefore, we posed following questions:

Q1: Is the Slovak PBQ reliable?

Q2: What are evidences of validity for the Slovak PBQ?

Q3: What is the factor solution for the Slovak PBQ?

\section{Methods}

Our sample consisted of 372 mothers, ages ranging from $18-44(\mathrm{M}=29.7, \mathrm{SD}=5.26)$. Both primiparous (56.7\%) and multiparous women were included. They gave birth in Slovakian hospitals. Data collection was carried out from September 2015 until March 2018. All participants completed the Postpartum bonding questionnaire and some of them other three tools.

The Postpartum Bonding Questionnaire (PBQ) (Brockington et al. 2001) is a 25-item screening instrument used to detect problems in bonding. Participants indicate agreement with the items on a six-point scale $(0=$ Not at all; $6=$ Always $)$ in four factors- Impaired Bonding, Rejection and Anger, Anxiety about care and Risk of Abuse. Summary indices are computed for each factor as well as for whole questionnaire (Lack of Bonding). Low scores denote good bonding. The PBQ was translated by one of us (KG, psychologist and translator into/to English) and Eva Szobiová (psychologist). Hence, we achieved consensus and administered items as a part of dissertation project. Since then, we have been collecting data to perform factorial analysis.

The Edinburgh Postnatal Depression Scale (EPDS, Cox et al., 1987) was developed to screen depressive symptoms in postpartum mothers. It is a simple self-assessment tool consisting of 10 items scored on a 4-point scale. High scores indicate more depressive symptoms.

The Depression, Anxiety, and Stress Scale (DASS, Lovibond \& Lovibond, 1995) consists of 42 items, divided into subscales of stress, anxiety, and depression, with a 4-point Likert scale. The items focus on physiological states as well as emotional experience. High scores show more symptoms of stress, anxiety, and depression.

18-item Parental stress scale (PSS, Berry \& Jones, 1995) is used to measure the level of stress in the non-clinical population including parental rewards, parental stressors, lack of control, and parental satisfaction. Items are evaluated on a 5-point Likert scale. Low scores indicate low level of parental stress.

\section{Results}

\subsection{Descriptive statistics for PBQ items}

In table 1 we present descriptive statistics for the PBQ items. Most of the items received low scores and were positively skewed and leptokurtic. Extreme points on the 5-point Likert scale are the most frequent modes except modes in three items. The internal consistency of all factors was assessed using Cronbach's alpha. Internal consistency is high for Lack of Bonding $(\alpha=.897)$ and varies in factors- $\alpha=.820$ for Impaired Bonding, $\alpha=.779$ for Rejection and Anger, $\alpha=.506$ for Anxiety about Care and $\alpha=.321$ for Risk of Abuse.

\subsection{Confirmatory factor analysis (CFA)}

Since in our previous research we found correlations among factors, we chose to perform confirmatory factor analysis (table 1). Both Kaiser-Meyer-Olkin measure of sampling adequacy (KMO .909) and Bartlett's test of sphericity $(\mathrm{p}<.001)$ were very good. $33.78 \%$ of the variance is explained by factor 1 ( 13 items), $7.98 \%$ by factor 2 ( 8 items), $4.04 \%$ by factor 3 ( 5 items), and $2.40 \%$ by 
factor 4 (2 items). Three items did not load on any factor and 6 items loaded on more than 1 factor. Eigenvalue of the first factor was at least 4-times greater than of the others. In table 2 we can see that the factors are moderately correlated, either in a positive or negative way.

Table 1. Item descriptive statistics, pattern matrix of CFA and rotated component matrix of PCA, source: authors.

\begin{tabular}{|c|c|c|c|c|c|c|c|c|c|c|c|c|c|c|}
\hline \multicolumn{9}{|c|}{} & \multicolumn{3}{c|}{$\begin{array}{c}\text { Confirmatory factor } \\
\text { analysis }\end{array}$} & \multicolumn{6}{c|}{ Principal component } \\
analysis
\end{tabular}

S-Skewness; K-Kurtosis; SD- standard deviation, $M$ - mean, SE-standard error of mean, F-factor, C-component, Factor analysisExtraction Method: Principal Axis Factoring. Rotation Method: Oblimin with Kaiser Normalization. Rotation converged in 11 iterations. Principal component analysis- Rotated component matrix, Extraction Method: Principal Component Analysis. Rotation Method: Varimax with Kaiser Normalization. Rotation converged in 7 iterations.

Table 2. Factor correlations matrix in confirmatory factor analysis, source: authors.

\begin{tabular}{|lcccc|}
\hline Factor & 1 & 2 & 3 & 4 \\
\hline 1 & 1.000 & & & \\
2 & .320 & 1.000 & & \\
3 & -.451 & -.230 & 1.000 & \\
4 & .304 & .428 & -.170 & 1.000 \\
\hline
\end{tabular}

\subsection{Principal component analysis (PCA)}

Given that the CFA did not suggest a good comparative fit with the previous factorial solution of the PBQ, we decided to perform PCA with varimax rotation to have the components independent (table 1). 6 components were extracted. Component 1 comprises 15 items that explained $35,58 \%$ of the variance with factor loadings from .306 up to .733. The component 1 was clearly dominant, with an eigenvalue more than at least four times greater than the eigenvalues of the other five components. $2^{\text {nd }}$ component explains $9.42 \%$ of the variance, $3^{\text {rd }}$ component $5.97 \%, 4^{\text {th }}$ component $4.65 \%, 5^{\text {th }}$ component $4.39 \%$, and $6^{\text {th }}$ component $4.03 \%$.

\subsection{New suggestions}

One of the solutions would be to keep one-factor solution and choose the best factor from CFA analysis. Factor 1 explains most of the variance (33.78\%) compared to other factors. It comprises 13 items (see table 4) and we could name this factor Distance. The internal consistency is high ( $\alpha .884)$.

We could also sort and manipulated with the items according to their wording and choose best items for each factor from PAF (bolded factor loadings in Table 2). Factor 1 thus comprises 12 items (PBQ1, PBQ2, PBQ3, PBQ4, PBQ5, PBQ8, PBQ9, PBQ11, PBQ13, PBQ17, PBQ22, PBQ23) with $\alpha=.875$ and we named it Distance and Regret. Factor 2 includes 5 items (PBQ7, PBQ10, PBQ14, PBQ19, PBQ21; $\alpha=.862$ ) and is characterized as Anger. Factor 3 describes feeling of Dislike and 4 items 
(PBQ15, PBQ16, PBQ21, PBQ24) are included $(\alpha=.783)$. The last factor 4 Uneasiness consists of 2 items (I PBQ12, PBQ25) with $\alpha=.680$. The items $(n=22)$ could be counted up since the internal consistency is high $(\alpha=.912)$. Factors correlation coefficient varies from .210 up to .577 , all statistically significant. Correlation of Uneasiness factor with Dislike was small, with Anger moderate and with other two strong.

Another solution is to keep the first component from PCA that consists of 15 items with explanative power of variance $(35,58 \%)$. This first component is the same as the first factor from CFA, but two items are added: PBQ6 (The baby doesn't seem to be mine.) and PBQ24 (I feel like hurting my baby.) We could name this component Distance and Regret as well. The internal consistency is high $(\alpha .880)$.

\subsection{Evidence for convergent validity}

Since the original factorial solution was not confirmed, we carried out correlations with summary index (Lack of Bonding) of the PBQ. We found weak and moderate relationship with depression (Edinburgh Postnatal Depression Scale, $r s=.251$; Depression Anxiety Stress scale-42 depression $r s=.404 ; n=79)$, moderate with stress and anxiety (Depression Anxiety Stress scale-42, stress $\mathrm{rs}=.392$; anxiety $\mathrm{rs}=.496 ; \mathrm{n}=79$ ) and strong with parental stress (Parental Stress Scale score; $\mathrm{rs}=.674$, $\mathrm{n}=99)$.

\section{Discussion}

The Slovak version of the Postpartum Bonding Questionnaire proved to have good overall reliability, but Cronbach $\alpha$ was low for two factors (Anxiety about care and Risk of abuse). Most of the items received low scores and were positively skewed and leptokurtic that has been confirmed in a general non-clinical sample in previous research (Greškovičová et al., 2018; Greškovičová \& Szobiová, 2016; Ohashi et al., 2016). The factor structure of the PBQ was examined using confirmatory factor analysis with principal axis method with oblimine rotation. We did not extract the original 4-factor solution of the PBQ which seem to be a general problem (Ghahremani et al., 2019; Reck et al., 2006; Wittkowski et al., 2010) could be associated with our non-clinical population and their positively skewed scores which happens in other studies as well (Ohashi et al., 2016). Secondly, Ohashi et al (2016) emphasizes that Brockington et al. (2001) underwent PCA which yields different results than CFA and, consequently, some items load on more than one factor which was also our case (6 items).

Our results instead suggest that the PBQ could have a 1 -factor solution. Factor 1 explains most of the variance $(33.78 \%)$ compared to other factors. It comprises 13 items and could be named as Distance and Regret because it deals with women's feelings toward her maternal role plus physical and emotional distance from the baby. The internal consistency is high $(\alpha .884)$. Very similar result was provided by PCA that we used to minimize the number of items and to produce a general factor with largest possible variance. Both factor 1 and component 1 explained more than $30 \%$ of variance and they differed just in two items (added in component 1) and their eigenvalue was at least 4-times higher than the rest of the factors/components. This suggest that there is one strong general factor underlying the PBQ.

We found evidences for the convergent validity of the lack of bonding in PBQ with parental stress (strong relationship), anxiety and stress (moderate relationship), and partly depression (small to moderate strength of the relationships).

Among limits we can see tools used for validity evidence. Only EPDS is usually used to provide the evidence and it is common to use other instrument measuring bonding. Since in Slovakia there is a gap in these instruments, we had to choose other ones. There is also a room to find evidence with other concepts, such as psychological types (Lisá, 2017) or traits (Lisá \& Kališ, 2019).

Sample without participants for clinical population could be another drawback. Some of the research is carried out with or without clinical sample. However, we must keep in mind that this instrument is originally designed as a screening instrument, so we feel a strong urge to perform these analyses in a clinical sample as well. Furthermore, we did not provide other reliability confirmation, such as consistency over time or other evidences of validity.

Taking these shortcomings into consideration, we advise to use the Slovak version of the PBQ as a tool to measure bonding in a research context and to use overall summary index (composite score) instead of original factorial solution. Women with lack of or lower intensity of bonding represent a risk group and it is also important to examine factor solution in different samples. Still, process of cross-cultural tools to measure bonding demonstrates the importance of evaluations of conceptual, item, semantic and operational equivalence. We see that lack of bonding is not the same as sufficiency of bonding and we should therefore have more tools to tap the "positive bonding". 


\section{Acknowledgments}

BZ and RF organized the data collection. KG contributed to the theory development, design, statistical analyses, and writing of the paper.

\section{References}

Berry, J. O., \& Jones, W. H. (1995). The Parental Stress Scale: Initial Psychometric Evidence. Journal of Social and Personal Relationships, 12(3), 463-472. https://doi.org/10.1177/0265407595123009

Brockington, I. F., Oates, J., George, S., Turner, D., Vostanis, P., Sullivan, M., Loh, C., \& Murdoch, C. (2001). A screening questionnaire for mother-infant bonding disorders. Archives of Women's Mental Health, 3(4), 133-140. https://doi.org/10.1007/s007370170010

Cox, J. L., Holden, J. M., \& Sagovsky, R. (1987). Detection of postnatal depression. Development of the 10-item Edinburgh Postnatal Depression Scale. The British Journal of Psychiatry: The Journal of Mental Science, 150, 782-786. https://doi.org/10.1192/bjp.150.6.782

Garcia-Esteve, L., Torres, A., Lasheras, G., Palacios-Hernández, B., Farré-Sender, B., Subir??, S., Vald??s, M., \& Brockington, I. F. (2016). Assessment of psychometric properties of the Postpartum Bonding Questionnaire (PBQ) in Spanish mothers. Archives of Women's Mental Health, 19(2), Article 2. https://doi.org/10.1007/s00737-015-0589-x

Ghahremani, S., Arian, H., Ghahremani, S., Rakhshanizadeh, F., Rahimi, R., \& Ghazanfarpour, M. (2019). Factorial Structures of Postpartum Bonding Questionnaire (PBQ): A Systematic Review. International Journal of Pediatrics, 7(4). https://doi.org/10.22038/ijp.2018.36262.3164

Greškovičová, K. (2016). Bonding a attachment v kontexte rodičovstva [Dissertation thesis, Paneuropean University].

https://opac.crzp.sk/?fn=detailBiblioForm\&sid=08B0A201CAF64A608886C6D1F450

Greškovičová, K., \& Szobiová, E. (2016). Bonding of women and men toward their infants. In L. Pitel (Ed.), Sociálne procesy a osobnost' 2016 (pp. 126-133). Ústav experimentálnej psychológie, Centrum spoločenských a psychologických vied SAV.

Greškovičová, K., Szobiová, E., \& Zdechovanová, B. (2018). Bonding toward infants in women and men- individual and pair analysis. In L. Sabová (Ed.), PSYCHOLOGICA XLVII (pp. 39-46). Stimul.

Lisá, E. (2017). Analysis of personality traits among psychological types. Ad Alta, 7(2), 118-122.

Lisá, E., \& Kališ, M. (2019). The psychometric characteristics of the Hexaco-100 questionnaire used in the selection and non-selection situation. Work and Organizational Psychology 2019: Proceedings of the 18th International Conference, 144-153.

Lovibond, S. H., \& Lovibond, P. F. (1995). Depression, Anxiety and Stress Scale-21 items (DASS-21)Scale-English. Manual for the Depression Anxiety \& Stress Scales, 1-2.

Ohashi, Y., Kitamura, T., Sakanashi, K., \& Tanaka, T. (2016). Postpartum Bonding Disorder: Factor Structure, Validity, Reliability and a Model Comparison of the Postnatal Bonding Questionnaire in Japanese Mothers of Infants. Healthcare, 4(3), 50. https://doi.org/10.3390/healthcare4030050

Perrelli, J. G. A., Zambaldi, C. F., Cantilino, A., \& Sougey, E. B. (2014). Mother-child bonding assessment tools* *Study conducted at Universidade Federal de Pernambuco, Recife, PE, Brazil. Revista Paulista de Pediatria (English Edition), 32(3), 257-265. https://doi.org/10.1016/S23593482(15)30020-8

Reck, C., Klier, C. M., Pabst, K., Stehle, E., Steffenelli, U., Struben, K., \& Backenstrass, M. (2006). The German version of the Postpartum Bonding Instrument: Psychometric properties and association with postpartum depression. Archives of Women's Mental Health, 9(5), 265-271. https://doi.org/10.1007/s00737-006-0144-x

Taylor, A., Atkins, R., Kumar, R., Adams, D., \& Glover, V. (2005). A new Mother-to-Infant Bonding Scale: Links with early maternal mood. Archives of Women's Mental Health, 8(1), 45-51. https://doi.org/10.1007/s00737-005-0074-z

van Bussel, J. C. H., Spitz, B., \& Demyttenaere, K. (2010). Three self-report questionnaires of the early mother-to-infant bond: Reliability and validity of the Dutch version of the MPAS, PBQ and MIBS. Archives of Women's Mental Health, 13(5), 373-384. https://doi.org/10.1007/s00737-009-0140-z

Wittkowski, A., Wieck, A., \& Mann, S. (2007). An evaluation of two bonding questionnaires: A comparison of the Mother-to-Infant Bonding Scale with the Postpartum Bonding Questionnaire in a sample of primiparous mothers. Archives of Women's Mental Health, 10(4), 171-175. https://doi.org/10.1007/s00737-007-0191-y

Wittkowski, A., Williams, J., \& Wieck, A. (2010). An examination of the psychometric properties and factor structure of the Post-partum Bonding Questionnaire in a clinical inpatient sample. British Journal of Clinical Psychology, 49(2), 163-172. https://doi.org/10.1348/014466509X445589 